\title{
Optimal Stem Identification in Presence of Suffix List
}

\author{
Vasudevan N and Pushpak Bhattacharyya \\ Computer Science and Engg Department \\ IIT Bombay, Mumbai \\ vasudevan@cse.iitb.ac.in, pb@cse.iitb.ac.in
}

\begin{abstract}
Stemming is considered crucial in many NLP and IR applications. In the absence of any linguistic information, stemming is a challenging task. Stemming of words using suffixes of a language as linguistic information is in comparison an easier problem. In this work we considered stemming as a process of obtaining minimum number of lexicon from an unannotated corpus by using a suffix set. We proved that the exact lexicon reduction problem is NP-hard and came up with a polynomial time approximation. One probabilistic model that minimizes the stem distributional entropy is also proposed for stemming. Performances of these models are analyzed using an unannotated corpus and a suffix set of Malayalam, a morphologically rich language of India belonging to the Dravidian family.
\end{abstract}

\section{Introduction}

Stemming is a crucial component in most of the NLP applications. Since the stemming identifies the same stem for all inflectional variants of a lexeme, it will improve the performance of information retrieval systems. Inflectional suffix in a word carries its morphosyntactic information and paradigm information. For example, by stemming the word boys, we will get the suffix s from that word. This suffix carries the information about its plurality. Such information is essential for many NLP applications like machine translation. This indicates the importance of building good stemmer for languages.

Building a morphology analyser or a stemmer is always a challenging task for all languages. This is more challenging for inflectional, agglutinative and isolated languages. Lot of linguistic expertise is needed for building such tools. This is again difficult for those languages which have no linguistic tradition. The linguistic expertise needed for stemming may not be available for such languages. So they have to rely on word forms in a corpus and their processing. Therefore building a low cost automatic stemmer from a corpus for such languages has a great significance.

Building a stemmer using only an unannotated corpus is the most inexpensive feasible approach. Since there is no availability of direct linguistic information and supervision, such a system with good performance is very difficult to build. 
Knowledge of inflectional suffixes in a language will reduce the level of difficulty. I.e. the performance of stemming using only an unannotated corpus can be improved by using a set of inflectional suffixes. Usually a language has a small closed set of inflectional suffixes. Identification of this suffix set of a language is a relatively easier job. So we consider a semi-supervised scenario where the suffix list is given. In this work we are trying to build a stemmer using an unannotated corpus with sufficiently large number of distinct words and a set of inflectional suffixes. Sample sets of inflectional suffixes in English and Malayalam are shown below (Example 1,2)

Example 1. English: $\{\mathbf{s}$, es, ed, ing, ... $\}$

Example 2. Malayalam: $\{$ ๓லீ $\{$ kal $\}$ (Plural), ๓ஃ $\{$ kale $\}$ (Plural + Accusative),

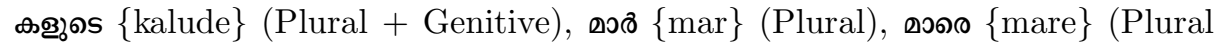

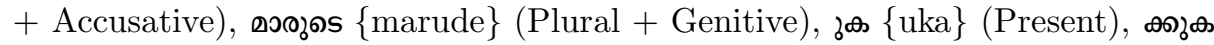
$\{$ kkuka\} (Present), १ \{i\} (Past), 子० $\{\mathrm{um}\}$ (Future), ... $\}$

Inputs of this stemming problem are an unannotated corpus (Corpus) and a suffix set (Suffixset), and the output is the stem of each word. A small input and its output is shown in Example 3.

Example 3. Input: Corpus $=\{$ mosses, moss, boys, boy $\}$, Suffixset $=\{\mathbf{e s ,} \mathbf{s}$, $\phi\}$, where $\phi$ is the null suffix.

\section{Output: $\{$ moss (mosses), moss (moss), boy (boys), boy (boy)\}}

We make an assumption that Suffixset includes all orthographic variations of all valid suffixes in the language. ఉ the variants of Malayalam ${ }^{1}$ plural marker $\boldsymbol{\infty} \boldsymbol{s}\{$ kal $\}$. So a Malayalam Suffixset contains all these variants. Some languages have only one possible suffix in a word, while others have more. In such languages a word has the structure

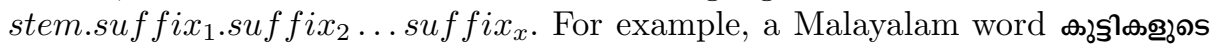
\{kuttikalude\} (child+Plural+Genitive) contains the stem क્ઠకీ \{kutti\} (child), a

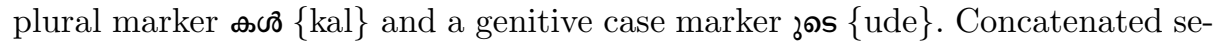
quence of suffixes, suffix $x_{1}$ suffix $x_{2} \ldots$ suffix $x_{x}$ is considered as a suffix in such words. So Suffixset of such a language contains some concatenations of suffixes

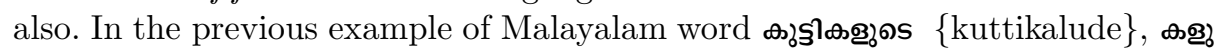
๑s $\{$ kalude $\}$ is actually a concatenation of suffixes क we consider this as a single suffix. We take another assumption that the Corpus contains sufficiently large number of words.

To define the stemming problem with suffix set, let us introduce a term possible_stem_set. possible_stem_set of a word $w$ be the set of all prefixes of $w$ such that $w$ can be expressed as the concatenation of that prefix and a suffix from Suffixset.

$$
\text { possible_stem_set }(w)=\{\text { st }: \exists x \in \text { Suffixset } \text { such that } w=\text { st. } x\}
$$

Consider the input shown in Example 3. possible_stem_set of each word in the Corpus is shown in the Table 1 . Now formally we can say, stemming is the 
Table 1: possible_stem_set and their Correct Stem

\begin{tabular}{lll}
\hline \hline word & possible_stem_set & correct stem \\
\hline \hline mosses & $\{$ mosses, mosse, moss $\}$ & moss \\
moss & $\{$ moss, mos $\}$ & moss \\
boys & $\{$ boy, boys $\}$ & boy \\
boy & $\{$ boy $\}$ & boy \\
\hline \hline
\end{tabular}

process of identifying $\operatorname{stem}(w)$ optimally for all word $w$, where $\operatorname{stem}(w)$ is an element of possible_stem_set $(w)$.

The stemming process is the selection of correct entry from possible_stem_set of each word in Corpus. In the previous example, the stemmer needs to select the stems moss from possible_stem_set(mosses), moss from possible_stem_set(moss), boy from possible_stem_set(boys) and boy from possible_stem_set(boy).

The roadmap of the paper is as follows. Related work of the stemming problem is briefly summarized in section 2 . We propose two models for stemming problem in this work. In section 3, we propose a deterministic model that reduces the number of distinct stems. In section 4 , we propose a probabilistic model that learns the distribution by reducing the entropy. A case study of these models in one of the morphologically rich language Malayalam is included in section 5. Performances of these models are measured in this section by using a wordlist and a suffix set.

\section{Related work}

Morphology learning is one of the widely attempted problem in the literature. A recent survey paper by Harald Hammarstrom [1] gives an overall view on unsupervised morphology learning. There are lots of probabilistic approaches for morphology learning. Linguistica model [2], maximum a posteriori model [3], stochastic transducer based model [4] and generative probabilistic model [5] are the relevant probabilistic models for stemming that we found in the current literature. A Markov Random Field by Dreyer [6] is also a useful work related to unsupervised morphology.

Graph based model [7], lazy learning based model [8], clustering based same stem identification model $[9,10]$ ParaMor system for paradigm learning [11] are also relevant works in the same area. Full morpheme segmentation and automatic induction of orthographic rules by Sajib Dasgupta $[12,13]$ is also a relevant work.

We never found any model which use the information from suffix list. To the best of our knowledge, this is the first attempt for stemming in presence of suffix list. We found that Linguistica model is the closely related approach to ours. Frequency of stem and suffix candidates plays a crucial role in Linguistica model. Linguistica model is an optimal stem identification model by reducing

\footnotetext{
${ }^{1}$ A morphologically rich language of India belonging to the Dravidian family.
} 
Table 2: Examples of valid_mapping

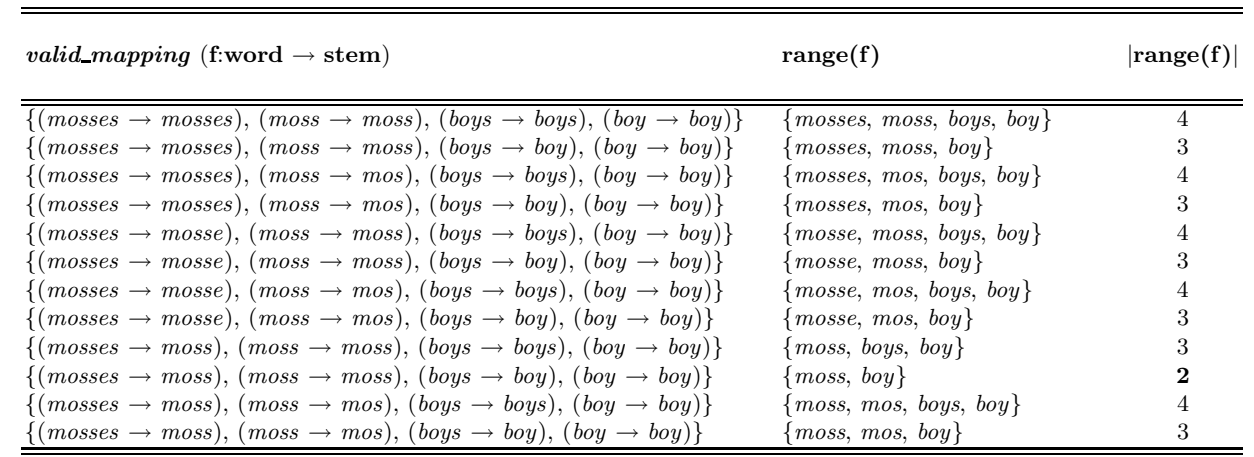

the description length. In this work, we minimizes the number of distinct stems and entropy of stem distribution.

\section{Minimum Stem Range (MSR) model}

Given the suffix set, stemming can be viewed as a process of reduction of lexicon entry. Minimum Stem Range (MSR) model is a direct and intuitive translation of this aspect of stemming problem to a well-defined computational model. MSR model finds out a mapping from each word to one of the string in its possible_stem_set. If suffix set is complete then actual stem of each word should be in possible_stem_set of that word. So, if possible_stem_set of a word contains exactly one entry then any mapping identifies the actual stem of that word. In the Table 1, possible_stem_set of boy contains only one element boy. So any mapping to possible_stem_set choose the correct stem boy from possible_stem_set(boy). Otherwise the actual stem needs to be identified by using the information from other words.

\subsection{Model}

MSR model is a computational model for the stemming problem. This model finds a mapping from each word in input corpus to its stem (a starting substring of the word). A mapping function $f$ from each word in input corpus to its stem (a starting substring of the word) is called as a valid_mapping if and only if each word in input corpus is gets mapped to one of the stems in its possible_stem_set. All valid_mappings from Example 3 are shown in Table 2 (possible_stem_sets of this sample corpus is shown in Table 1). A mapping (mosse, mos, boys, boy) means, first word mosses is gets mapped to mosse, second word moss is gets mapped to mos, boys is gets mapped to boys and boy is gets mapped to boy.

MSR model will find out a valid_mapping with minimum range. Lets define the MSR model formally. 
Input: Corpus (a sufficiently large list of plain words) and Suffixset (set of all suffixes)

Output: A valid_mapping, $f^{*}$ with minimum cardinality of range,

$$
f^{*}=\underset{f \in \text { valid_mapping }}{\text { argmin }}\{\mid \text { range }(f) \mid\}
$$

Out of these 12 valid_mappings shown in Table 2, (moss, moss, boy, boy) have the minimum range. So the MSR model will identify this mapping.

Theorem 1. If a language does not have any morphological ambiguity and MSR model identifies a valid stem for at least one word from all group of words with same stem then MSR model will identify the correct stem from all words.

Proof. Lets assume there is no morphological ambiguity. So there will be exactly one split that generates a valid stem and a valid suffix. If one of the valid stem is in possible_stem_set of a word then that will be the correct stem of that word. Otherwise there will be more than one way to split that word and that will be a morphological ambiguity. So if MSR problem identifies a valid stem from a word then that will be the correct stem of that word.

Suppose there are $m$ groups of words with the same stem. There exist a valid_mapping with range of size exactly $m$ by correctly identifying all correct stems. So the range of output of MSR model will be less than or equal to $m$.

Let $f$ be the output of MSR model that identifies correct stem from at least one word from all $m$ groups. So, all valid stems from all words in input corpus will be in the range of $f$. Since there exist exactly one valid stem in each possible_stem_set, any incorrect valid_mapping produces an invalid stem. So if there is any incorrect mapping, then the range of $f$ will be greater than $m$. If there is any incorrect mapping in $f$ then $f$ will not be the output of MSR model. Therefore the MSR model will identify the correct stem from all words.

Theorem 2. Problem of computing MSR model (MSR problem) is NP-Hard

Proof. To prove the MSR problem is NP-hard, we want to find a polynomial time reduction from an existing NP-hard problem to MSR problem. Let us take minimum vertex cover problem, a known NP-hard problem for the reduction. Input of minimum vertex cover problem is an undirected graph $G=(V, E)$ and output is a set of vertices (vertex cover) $V C$ with minimum cardinality, where $V C \subseteq V$ and for every edge $e_{i j}$ in $E$, either $v_{i} \in V C$ or $v_{j} \in V C$. Given an instance of vertex cover problem $(G=(V, E))$, we construct an instance to MSR problem (Corpus, Suffixset) as follows.

Suppose $G=(V, E), V=\left\{v_{i}\right\}, E=\left\{e_{i j}\right\} 1 \leq i, j \leq n$ be the input of vertex cover problem. For every edge $e_{i j}$, without loosing generality we can say $i \leq j$. For each edge $e_{i j}$ add a word $c_{1} . c_{2} \ldots c_{j} . m_{i j}$ in to the Corpus. Here $c_{1}, c_{2}, \ldots c_{j}$ and $m_{i j}$ can be any distinct characters. For each edge $e_{i j}$ add $c_{i+1} \ldots c_{j} . m_{i j}$ and $m_{i j}$ in to the Suffixset (if $i=j$ then add only $m_{i j}$ in to the Suffixset). 
Consider a small graph shown below. Corresponds to four edges $\left(e_{12}, e_{13}, e_{23}, e_{34}\right)$ add four words to the Corpus. Similarly corresponds to four edges add eight suffixes to the Suffixset. These words and suffixes are shown in the second and third columns of Table 3 .

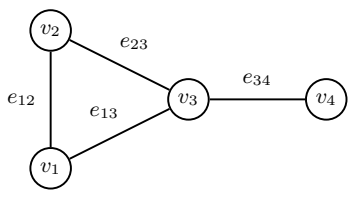

Table 3: Reduction Example of the Graph

Figure 1: Example Graph to Elucidate Re-

\begin{tabular}{llllll}
\hline \hline & word list & suffix list & possible_stem_set & $f$ & VC \\
\hline \hline$e_{12}$ & $c_{1} c_{2} m_{12}$ & $c_{2} m_{12}, m_{12}$ & $\left\{c_{1}, c_{1} c_{2}\right\}$ & $c_{1}$ & $v_{1}$ \\
$e_{13}$ & $c_{1} c_{2} c_{3} m_{13}$ & $c_{2} c_{3} m_{13}, m_{13}$ & $\left\{c_{1}, c_{1} c_{2} c_{3}\right\}$ & $c_{1}$ & $v_{1}$ \\
$e_{23}$ & $c_{1} c_{2} c_{3} m_{23}$ & $c_{3} m_{23}, m_{23}$ & $\left\{c_{1} c_{2}, c_{1} c_{2} c_{3}\right\}$ & $c_{1} c_{2} c_{3}$ & $v_{3}$ \\
$e_{34}$ & $c_{1} c_{2} c_{3} c_{4} m_{34}$ & $c_{4} m_{34}, m_{34}$ & $\left\{c_{1} c_{2} c_{3}, c_{1} c_{2} c_{3} c_{4}\right\}$ & $c_{1} c_{2} c_{3}$ & $v_{3}$
\end{tabular}
duction Procedure

Let $f$ be the output of MSR problem and $R$ is the range of $f$. Since all $c_{i}$ and $m_{i j}$ are distinct, possible_stem_set $\left(c_{1} \ldots c_{j} . m_{i j}\right)$ will be $\left\{c_{1} \ldots c_{j}, c_{1} \ldots c_{i}\right\}$. See the possible_stem_set of each word in the previous example shown in Table 3. So each string in $R$ will be in the form of $c_{1} \ldots c_{k}$ for some $k$. For each such string, add the vertex $v_{k}$ in to $V C$. Here the cardinality of $R$ and $V C$ will be same. In the example the corresponding vertex cover (VC) of $f$ function is a minimal vertex cover $\left(\left\{v_{1}, v_{3}\right\}\right)$.

To prove the correctness of reduction we want to show the solution of MSR problem will be same as the solution of minimum vertex cover problem.

Let say $f^{*}$ is the solution of MSR problem and $V C^{*}$ is the corresponding output instance of minimum vertex cover problem. For each word $c_{1} \ldots c_{j} . m_{i j}$ either $c_{1} \ldots c_{j}$ or $c_{1} \ldots c_{i}$ will be in the range of any valid_mapping. Therefore $V C^{*}$ will be a valid vertex cover and that can be the solution of minimum vertex cover problem. Suppose $V C^{*}$ is not the solution of minimum vertex cover problem, then there exist another valid vertex cover $V C^{\prime}$, such that $\left|V C^{\prime}\right|<$ $\left|V C^{*}\right|$. Then for $V C^{\prime}$ we can define a valid_mapping, $f^{\prime}$,

$$
f^{\prime}\left(c_{1} c_{2} \ldots c_{j} m_{i j}\right)=\left\{\begin{array}{l}
c_{1} c_{2} \ldots c_{i} \text { if } v_{i} \text { is in } V C^{\prime} \\
c_{1} c_{2} \ldots c_{j} \text { otherwise }
\end{array}\right.
$$

Here we can see $\left|\operatorname{range}\left(f^{\prime}\right)\right| \leq\left|V C^{\prime}\right|$

$\Rightarrow\left|\operatorname{range}\left(f^{\prime}\right)\right|<\left|V C^{*}\right|$

$\Rightarrow\left|\operatorname{range}\left(f^{\prime}\right)\right|<\left|\operatorname{range}\left(f^{*}\right)\right|$

By the definition of $f^{*}$ this is a contradiction. Therefore $V C^{*}$ always will be the solution of minimum vertex cover problem.

Let $V C^{*}$ is the solution of minimum vertex cover problem. Now we can define a corresponding valid_mapping $f^{*}$ for $V C^{*}$,

$$
f^{*}\left(c_{1} c_{2} \ldots c_{j} m_{i j}\right)=\left\{\begin{array}{l}
c_{1} c_{2} \ldots c_{i} \text { if } v_{i} \text { is in } V C^{*} \\
c_{1} c_{2} \ldots c_{j} \text { otherwise }
\end{array}\right.
$$


Here $\left|V C^{*}\right| \geq\left|\operatorname{range}\left(f^{*}\right)\right|$. If $\left|V C^{*}\right|>\left|\operatorname{range}\left(f^{*}\right)\right|$ then there exist another vertex cover with less cardinality. But $V C^{*}$ is the minimum vertex cover. So $\left|\operatorname{range}\left(f^{*}\right)\right|$ will be same as $\left|V C^{*}\right|$.

Suppose $f^{*}$ is not the solution of MSR problem, then there will be another valid_mapping $f^{\prime}$ such that $\left|\operatorname{range}\left(f^{\prime}\right)\right|<\left|\operatorname{range}\left(f^{*}\right)\right|$. Then there will be a valid vertex cover $V C^{\prime}$ corresponds to $f^{\prime}$, s.t. $\mid$ range $\left(f^{\prime}\right)|=| V C^{\prime} \mid$

$$
\begin{aligned}
& \Rightarrow\left|V C^{\prime}\right|<\left|\operatorname{range}\left(f^{*}\right)\right| \\
& \Rightarrow\left|V C^{\prime}\right|<\left|V C^{*}\right|
\end{aligned}
$$

But $V C^{*}$ is the minimum vertex cover. So such a valid vertex cover does not exist. Therefore $f^{*}$ will be a solution of MSR problem.

\subsection{Approximation}

Since the MSR problem is NP-hard, it is difficult to find stems from a large data. In order to solve the stemming problem computationally, an approximation of the above model is required. Similarity of this model to set cover problem can be utilized to find an approximation algorithm. MSR problem can be reduced to set cover problem. Input of set cover problem is a set $U$ and a set of subsets $(S)$ of $U$ such that, $\bigcup_{s \in S} s=U$. Output of set cover problem is a subset of $S$, say $C$, such that $\bigcup_{s \in C} s=U$.

Let $S t$ be the set of all possible stems, i.e. $S t=\bigcup_{w \in \text { Corpus }}$ possible_stem_set $(w)$. Let $W(s t)$ is the set of words in Corpus where st is an element of its possible_stem_set, i.e. $W(s t)=\{w:$ st $\in$ possible_stem_set $(w)\}$. Take the sample input corpus $\{$ mosses, moss, boys, boy $\}$ and input suffix set $\{\mathbf{e s}, \mathbf{s}, \phi\}$. Set of all possible stems and its corresponding $W(s t)$ are shown in the Table 4 . Now consider the Corpus as $U$ and the set $\{W(s t)\}$ as $S$ of set cover problem. Here $\bigcup_{s t \in S t} W(s t)=$ Corpus. The output of this set cover problem is a set of stems $S t^{\prime}$ such that, for any word in Corpus, there exist at least one possible stem of that word in $S t^{\prime}$. By choosing any one possible stem of each word from $S t^{\prime}$, we can find a solution of MSR problem.

\begin{tabular}{|c|c|c|c|c|c|c|}
\hline Stem & mosses & mosse & moss & mos & boys & boy \\
\hline 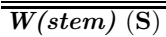 & \{mosses $\}$ & $\overline{\langle\text { \{mosses, moss }\}}$ & $\overline{\text { \{moss }\}}$ & 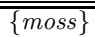 & "בboys $\}$ & $\overline{\langle\{\text { boys, boy }\}}$ \\
\hline
\end{tabular}

Table 4: Possible Stems and their W() Set

Any approximation algorithm of set cover problem can be directly used for this stemming problem. Best-known approximation of set cover problem is the greedy algorithm. The greedy algorithm choose subsets one by one from $S$ that have maximum number of uncovered elements in $U$, and cover those uncovered elements. The complexity of approximation algorithm is $O(N M)$ and approximation factor is $\log (N)$, where $N$ is the number of words in corpus and $M$ is the number of suffixes in suffix set. 


\section{Minimum Stem Entropy (MSE) model}

The MSR model and its approximation are deterministic approaches for the stemming problem. Greedy approximation of the above model may not find optimum mapping. Here we propose a new model, Minimum Stem Entropy (MSE) model, which is a probabilistic approach for the stemming problem.

\subsection{Model}

The probabilistic model assumes an uncertainty to choose the correct stem from possible_stem_set of each word. Input of MSE model is same as that of MSR model. Output of this model is a conditional probability distribution of stem given word $(\operatorname{Pr}(s t \mid w))$. $\operatorname{Pr}(s t \mid w)$ is the probability that string st (from possible stem set of $w, \operatorname{PSS}(w))$ is the stem of word $w$. From the $\operatorname{Pr}(s t \mid w)$ we can select the maximum probable stem candidate as the correct stem of that word, i.e. stem of a word $w, \operatorname{Stem}(w)=\operatorname{argmax}_{s t}\{\operatorname{Pr}(s t \mid w)\}$. Two basic conditions for $\operatorname{Pr}(s t \mid w)$ are,

$$
\begin{aligned}
& \operatorname{Pr}(s t \mid w) \geq 0 \text { for all } s t \text { in possible stem set of } w \\
& \sum_{s t \in P S S(w)}\{\operatorname{Pr}(s t \mid w)\}=1
\end{aligned}
$$

Many such probability distributions are possible. Most suitable distribution based on input corpus needs to be learned. The MSE model selects the distribution that minimizes the entropy of stem distribution. A stem distribution $\operatorname{Pr}(s t)$ is the probability of $s t$ is to be identified as correct stem of a randomly chosen word. We can write the stem distribution in terms of $\operatorname{Pr}(s t \mid w)$ as,

$$
\operatorname{Pr}(s t)=\sum_{w \in \text { corpus }}\{\operatorname{Pr}(w) \times \operatorname{Pr}(\text { st } \mid w)\}
$$

The MSE problem can be written as an optimization problem. I.e.

$$
\begin{aligned}
& \operatorname{Pr}(s t \mid w)=\underset{\operatorname{Pr}(\text { st } \mid w)}{\operatorname{argmin}}\{\operatorname{Entropy}(\operatorname{Pr}(s t))\} \\
& =\underset{\operatorname{Pr}(s t \mid w)}{\operatorname{argmin}}\left\{\operatorname{Entropy}\left(\sum_{w \in \text { corpus }}\{\operatorname{Pr}(w) \times \operatorname{Pr}(s t \mid w)\}\right)\right\} \\
& =\underset{\operatorname{Prgmin}(\operatorname{st} \mid w)}{\operatorname{argmin}}\left\{-\sum_{s t}\left\{\sum_{w}\{\operatorname{Pr}(s t \mid w) \times \operatorname{Pr}(w)\} \times \log \left(\sum_{w}\{\operatorname{Pr}(s t \mid w) \times \operatorname{Pr}(w)\}\right)\right\}\right\}
\end{aligned}
$$

MSE learns the probability of possible stem of each word that minimize the entropy of complete stem distribution. A stem distribution with low entropy has a tendency for a small stem set. So this model has a similarity with MSR model. With minimum stem distributional entropy, probability of a string is to be a correct stem in a randomly chosen word is either low or high. I.e. a string can easily classify in to a valid stem class or invalid stem class. So the process tries 
to learn the information about stems. The learning process identify the stem distribution such that, maximum information about stem is available from the input corpus. The formal definition of this model is shown below.

Input: Corpus (a sufficiently large list of plain words) and Suffixset (set of all suffixes)

Output: $\operatorname{Pr}(s t \mid w)$ for all $w$ in Corpus and st in possible_stem_set $(w)$

Consider a small corpus $\{$ mosses, moss $\}$ and a suffix set $\{\mathbf{e s}, \mathbf{s}, \phi\}$, where $\phi$ is the null suffix. The possible_stem_set of mosses is $\{$ mosses, mosse, moss $\}$ and possible_stem_set of moss is $\{$ moss, mos $\}$. Here the word probability can be taken as uniform distribution. So,

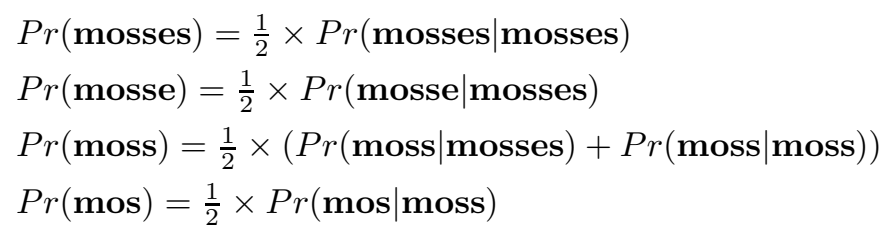

In this case, for $\operatorname{Pr}(\mathbf{m o s s} \mid \mathbf{m o s s e s})=1$ and $\operatorname{Pr}(\mathbf{m o s s} \mid \mathbf{m o s s})=1$, will get a zero stem distributional entropy. So the MSE will converge to this distribution, and the string moss will be selected as the stem of both mosses and moss.

\subsection{Methodology}

To learn the model from a corpus and a suffix set, an optimization problem needs to be solved. Since the objective function is not convex, an iterative hill climbing like approach is used. We used the Frank-Wolfe algorithm [14] to solve the optimization.

In each iteration of Frank-Wolfe algorithm, we solved a linear program. By converging to local optima, the algorithm will find out the best probability distribution given the input corpus and suffix set. Most probable stem from each word in corpus is then identified.

\section{Case study - Malayalam}

Malayalam is a Dravidian language spoken by 32 million people primarily in Kerala, a state in southern India [15]. Malayalam is highly agglutinative and inflectionally rich with a free word order. This language has a strong postpositional inflection. A Malayalam noun can be inflected for case, number, person and gender,

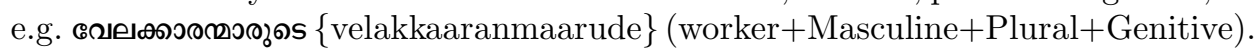
Verb can be inflected by suffix for mood, aspect and tense, e.g. வกஸा \{paranju\}

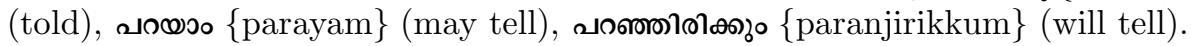

Approximated MSR model and MSE model are evaluated on Malayalam. We used a Malayalam unannotated corpus of size around 20000 words and a suffix 
set of around 200 Malayalam suffixes. We are extracted these words from the web. Malayalam is used by less than $0.1 \%$ of all the websites. Our assumption is that, the wordlist is sufficiently large so that it contains all morphological variants of words. In practical case this assumption may not hold. To reduce the gap between the ideal case and the real case, we make sure that, the training wordlist contains at least one more inflectional variant for each word. So we manually added such inflectional variants to wordlist if necessary.

We used the Morfessor [3] system to get a ballpark accuracy. We trained the Morfessor 1.0 model using their script downloaded from www.cis.hut.fi/projects/ morpho/morfessor1.0.perl with default arguments. The Morfessor score is around $17 \%$ only. Since Morfessor is not using any information from suffix set, a direct comparison between the Morfessor score and our scores is meaningless. Since we are unable to find any other comparable approaches for this problem from the literature, we want to constructed baseline models to check the significance of these proposed models.

The problem is about selecting correct entry from possible stem set of each word. We considered different trivial strategies to select the stem from possible stem set. One basic information is that a stem is a prefix of the word by stripping some valid suffix. One trivial approach that uses only this basic information is a random selection from possible stem set. We considered this as one of the baselines. Length of the suffix (or stem) is another easily available information that can use for stemming. Based on this information we can build two simple strategies, smallest stem or largest stem. We want to choose one of these approaches. Since we are doing experimentation on data, we decided to consider both approaches for experimentation and decide based on the scores. So we considered these two approaches as second and third baselines. Performances of two new proposed models and these three baseline models are evaluated using around 1500 Malayalam Unicode words from wordlist. The accuracies are shown in the Table 5 .

Table 5: Stemming Accuracies and Comparison with Baseline;

\begin{tabular}{|l||l|l|l|l|l|}
\hline \hline Model & $\begin{array}{l}\text { Baseline-1 (Min } \\
\text { Stem length) }\end{array}$ & $\begin{array}{l}\text { Baseline-2 (Max } \\
\text { Stem length) }\end{array}$ & $\begin{array}{l}\text { Baseline-3 (Ran- } \\
\text { dom selection) }\end{array}$ & Approx. MSR & MSE \\
\hline \hline Accuracies (20K words) & $84.58 \%$ & $20.09 \%$ & $44.20 \%$ & $91.32 \%$ & $\mathbf{9 3 . 9 1 \%}$ \\
\hline \hline
\end{tabular}

The results shown in the above table indicates the correctness and significance of proposed models for Malayalam. It also shows that MSE model slightly outperforms the Approx-MSR model. From the scores it is clear that, choosing the stem with minimum length is more suitable for Malayalam. If a word in a morphologically rich language ends with a valid suffix, most likely that will be its suffix. In agglutinative languages, more than one suffix can attach to a stem. So a word may ends with more number of valid suffixes. In this case the actual stem is the prefix by stripping largest suffix from the word. Since Malayalam is a morphologically rich and agglutinative language, the high accuracy of smallest 
stem baseline compared to largest stem baseline is very intuitive. Also note that, this may not be true for morphologically poor languages.

To get more insight about the shortcomings of these experimentations and models, we did an error analysis of two newly proposed systems. Each and every wrong splits are analyzed. Errors in both models are common and it follows same distribution. The errors in the models are mainly three types. These errors are as follows.

1. Because of the incomplete suffix set, the models may unable to split any other inflections of some words. So the possible_stem_set of such words will be totally independent from possible_stem_set of other words. Then our stemming models choose one stem from possible_stem_set of such words randomly and that may leads to wrong stemming.

2. Some orthographically similar words with different stem affect the stemming

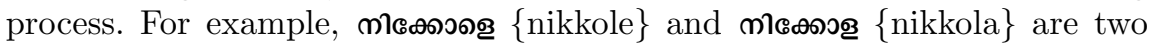
different proper nouns, but it looks very similar. By removing one of the

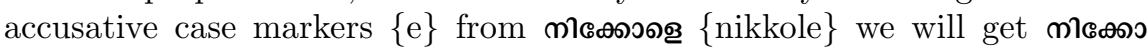

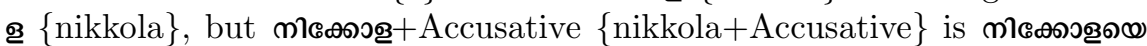
\{nikkolaye\}. In this case our stemming models wrongly selects the word

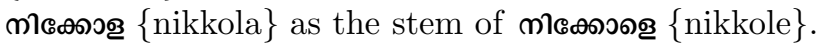

3. There may exist multiple solutions that minimize the number of distinct stems or stem distributional entropy. In such cases, our models randomly choose one of the solutions. That solution may have some wrong stems.

Some wrong stems identified by our models and its error type (3 types mentioned above) are shown in the Table 6 .

Table 6: Erroneous Stems Samples Found During Error Analysis

\begin{tabular}{|c|c|c|c|}
\hline Word & Stem (Identified) & Stem (Correct) & Error Type \\
\hline 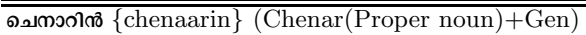 & لدMonிณ̋ \{chenaarin $\}$ & لدهMo $\{$ chenaa $\}$ & 1 \\
\hline 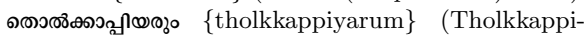 & 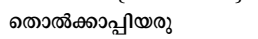 & 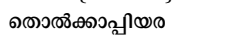 & 1 \\
\hline yar(Proper noun)+Conj) & $\{$ tholkkappiyaru $\}$ & $\{$ tholkkappiyara $\}$ & \\
\hline ભேm $\{$ vendi $\}$ (for that) & வேஸs $\{$ venda $\}$ & ભேஸा $\{$ vendi $\}$ & 1 \\
\hline றிகேேைஃ $\{$ nikkole $\}$ (Nikkole(Proper noun)) & ๓ிகேஸ๐ \{nikkola $\}$ & mிఱே๐๐ஃ $\{$ nikkole $\}$ & 2 \\
\hline ๑ిల్ఠณ̊ \{millan\} (Millan(Proper noun)) & هาอู $\{$ milla $\}$ & هిల్ఠm $\{$ millana $\}$ & 3 \\
\hline 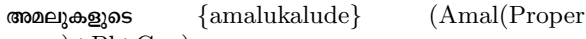 & 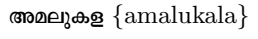 & สтவе $\{$ amala $\}$ & 1 \\
\hline noun $)+\mathrm{Pl}+\mathrm{Gen})$ & & & \\
\hline
\end{tabular}

One of the reason for the remaining errors are the incomplete suffix set. If the suffix set is not complete then stems of some words in the input corpus will not be identified. Stem information from such words may useful in the process of stem identification of other words.

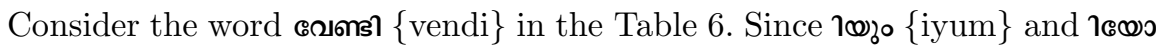

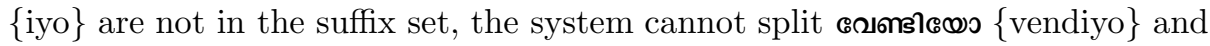

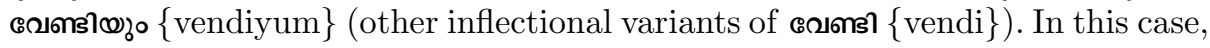
effectively there is no other word in the corpus for the splitting of the word 묙 \{vendi\}. So a string from possible_stem_set of ৫ேแ3 \{vendi\} selects randomly. 
Majority of remaining errors are because of this problem. So completion of the suffix set is considered for further improvement.

\section{Conclusion and Future Work}

To solve the stemming problem by using an unannotated corpus and a suffix set, two models are proposed. Problem of computing first model that directly reduces the number of lexicon entries is NP-hard. A greedy approximation for this model is also proposed. Second model is a probabilistic model and it reduces the entropy of stem distribution. Approximated version of first model and second model are evaluated on Malayalam corpus. We got the best accuracy of $93 \%$ by using the MSE model. Improvement in suffix set is proposed for future work. Analysing the performances of these proposed models in other languages is also considered for future work.

\section{References}

1. Hammarström, H., Borin, L.: Unsupervised learning of morphology. CL (2011) 309-350

2. Goldsmith, J.A.: Unsupervised learning of the morphology of a natural language. CL (2001) 153-198

3. Creutz, M., Lagus, K.: Unsupervised models for morpheme segmentation and morphology learning. TSLP 4 (2007)

4. Clark, A.: Partially supervised learning of morphology with stochastic transducers. In: NLPRS. (2001) 341-348

5. Snover, M.G., Jarosz, G.E., Brent, M.R.: Unsupervised learning of morphology using a novel directed search algorithm: taking the first step. In: Proc. of ACLWMPL-02. (2002) 11-20

6. Dreyer, M., Eisner, J.: Graphical models over multiple strings. In: Proc. of EMNLP-09. (2009) 101-110

7. Johnson, H., Martin, J.: Unsupervised learning of morphology for english and inuktitut. In: Proc. of NAACL-HLT-03. (2003) 43-45

8. van den Bosch, A., Daelemans, W.: Memory-based morphological analysis. In: Proc. of ACL-99. (1999)

9. Hammarström, H.: A naive theory of affixation and an algorithm for extraction. In: Proc. of HLT-NAACL-06. (2006) 79-88

10. Hammarström, H.: Poor man's stemming: Unsupervised recognition of same-stem words. In: AIRS. (2006) 323-337

11. Monson, C., Carbonell, J.G., Lavie, A., Levin, L.S.: Paramor and morpho challenge 2008. In: CLEF. (2008) 967-974

12. Dasgupta, S., Ng, V.: High-performance, language-independent morphological segmentation. In: HLT-NAACL. (2007) 155-163

13. Dasgupta, S., Ng, V.: Unsupervised morphological parsing of bengali. Language Resources and Evaluation (2006) 311-330

14. Lawphongpanich, S.: Frank-wolfe algorithm. In: Encyclopedia of Optimization. (2009) 1094-1097

15. David, S.M.I.P.S.: A morphological processor for malayalam language. Technical report, South Asia Research (2007) 